\title{
Determination of Topology Skeleton of Magnetic Fields in a Solar Active Region*
}

\author{
Hui Zhao ${ }^{1}$, Jing-Xiu Wang ${ }^{1}$, Jun Zhang ${ }^{1}$, Chi-Jie Xiao ${ }^{1}$ and Hai-Min Wang ${ }^{2}$ \\ ${ }^{1}$ National Astronomical Observatories, Chinese Academy of Sciences, Beijing 100012; \\ wangjx@bao.ac.cn \\ 2 Big Bear Solar Observatory, 40386 North Shore Lane, Big Bear City, CA 92314, USA
}

Received 2007 ; accepted 2007

\begin{abstract}
The knowledge of magnetic topology is the key to understand magnetic energy release in astrophysics. Based on observed vector magnetograms, we have determined threedimensional (3D) topology skeleton of the magnetic fields in active region NOAA 10720. The skeleton consists of six 3D magnetic nulls and a network of corresponding spines, fans, and null-null lines. For the first time, we have identified a spiral magnetic null in Sun's corona. The magnetic lines of force twisted around the spine of the null, forming a 'magnetic wreath' with excess of free magnetic energy and resembling observed brightening structures at extraultraviolet (EUV) wavebands. We found clear evidence of topology eruptions which are referred to as the catastrophic changes of topology skeleton associated with a coronal mass ejection (CME) and an explosive X-ray flare. These results shed new lights in exploring the structural complexity and its role in explosive magnetic activity. In solar astrophysics and space science, the concept of flux rope has been widely used in modelling explosive magnetic activity, although their observational identity is obscure or, at least, lacking of necessary details. The current work suggests that the magnetic wreath associated with the 3D spiral null is likely an important class of the physical entity of flux ropes.
\end{abstract}

Key words: Sun: magnetic fields — Sun: solar corona — Sun: activity

\section{INTRODUCTION}

The self-closure and frozen-in condition of magnetic fields in astrophysical and space plasmas make the electromagnetic interaction extremely complicated in the sense that the magnetized plasma is divided into distinct topologies. The explosive release of stored magnetic energy, which appears presumably in the topology interface, can not be realized without topology collapse. Solar active regions (ARs) represent a typical example of electromagnetic interaction in astrophysics. They are characterized by strong magnetic fields with complex topology, serving as a paradigm in astrophysics and space science. Since late 1940s it has been recognized that under certain conditions the breakdown of magnetic topology could produce solar flares and cosmic ray particles (Giowanelli 1946; Sweet 1958). To explore the magnetic skeleton in an AR, which comprises magnetic nulls (neutral points) and a network of spines ( $\gamma$ lines) and fans ( $\Sigma$ surfaces) (Lau \& Finn 1990; Parnell et al. 1996), is fundamental in understanding explosive solar activity (Priest et al. 1997). This, in turn, will guide to a thorough understanding of ubiquitous magnetic activity in the universe.

The 3D topology skeleton of Sun's magnetic fields, which is composed of nulls, spines, fans and separators (Priest et al. 1997), has only been described either by simple analytical models (Lau 1993; Brown \&

* Supported by the National Natural Science Foundation of China. 
Priest 2001; Parnell 2007), or by theoretical calculations with 'magnetic charges' or 'dipoles' to approximate the observed magnetic fields on solar surface (Seehafer 1986; Gorbachev \& Somov 1988; Démoulin et al. 1992; Longcope \& Klapper 2002; Longcope 2005). Comparing brightness observations with the above conceptual models, Filippov (1999), Aulanier et al. (2000), Fletcher et al. (2001), Maia et al. (2003) and $\mathrm{Li}$ et al. (2006) identified 3D nulls in the corona. These approaches have demonstrated that there, indeed, exist 3D magnetic nulls with spines and fans in the corona, forming a skeleton of magnetic topology. In addition, the magnetic energy release in solar flares is closely associated with these topology structures. However, as too many simplifications are adopted, e.g., the representation of the observed fields by discrete 'magnetic charges' or 'dipoles' and the assumption of the current-free (potential) fields, we are far from being able to evaluate current status of our knowledge about the real magnetic skeleton, let alone the nature of 3D magnetic reconnection in the solar atmosphere (Parnell 2007). Only recently, 3D magnetic nulls are identified in geomagnetotail based on the in situ measurements with Cluster spacecraft (Xiao et al. 2006). On the other hand, separatrices can also be approached by discontinuities in the footpoint mapping which is defined in a continuous magnetic boundary (Low 1987; Low \& Wolfson 1988). This discontinuities ascribe coronal nulls or bald patches (Seehafer 1986; Titov et al. 1993; Bungey et al. 1996). Some authors (Priest \& Démoulin 1995; Démoulin et al. 1996; Titov et al. 2002) give out measurement of mapping distortion or squashing to accurately locate the so-called quasi-separatrix layer (QSL). The QSL method is not a direct way to determine the separatrices. So it would take more time even than magnetic charge topology (MCT) method (Longcope 2005) to approach the same singular feature. From the footpoint mapping of magnetic boundary we also can not get clear skeleton as the methods depart from the nulls. But to study the features in photosphere or lower coronal, it is still a useful tool for its precise measurement of the discontinuities of the field lines.

The solid advances in the measurements of solar vector magnetic fields in recent years have open a possibility to observationally determine the topology skeleton in the Sun's active corona. In this paper, based on high quality and high cadence vector magnetograms and theoretical calculations, we have completely determined the topology skeleton of magnetic fields for a solar AR, NOAA 10720. The determined topology skeleton and its temporal evolution are approached, then, the explosive magnetic energy release in a major solar flare/CME event is understood with the knowledge of topology skeleton.

This paper is organized as followings: Section 2 is devoted to a description of observations of vector magnetic fields in AR 10720; Section 3 is a brief introduction of the methods used in this work to determine the topology skeleton; Section 4 is main results of the study on the determined topology skeleton and its temporal evolution which led to the major flare and CME activity; In Section 5, we present a particular discussion on the possible uncertainty of the results; Conclusions are finally summarized in the last section.

\section{OBSERVATIONS OF VECTOR MAGNETIC FIELDS IN AR 10720}

NOAA AR 10720 is a super AR which has created the largest and hardest proton flux $(>100 \mathrm{MeV})$ since 1989 and disastrous condition in space weather. It rotated onto the solar disk as a simple beta magnetic sunspot on January 10 and ended as a large, magnetic complex sunspot region on January 22, 2005. It grew rapidly and showed impressive activity while it transited the solar disk. During January 14-21 it produced 5 X-class flares and $18 \mathrm{M}$-class flares. Vector magnetic fields were mapped using Huairou Solar Observing Station (HSOS) vector-magnetograph (Ai and Hu 1986; Wang et al. 1996) from January 12 to 20, which provide clues in understanding the detailed magnetic evolution associated with major flares. Big Bear Solar Observatory (BBSO) obtained vector magnetograms in January 14-15 with high cadence of 1-2 minutes and high resolution around $1-2^{\prime \prime}$. The vector field evolution, thusly, could be traced in a round-the-clock way from January 14-16.

The BBSO vector magnetograms were obtained by Digital Vector Magnetograph (DVMG) system, covering an area of about $300^{\prime \prime} \times 300^{\prime \prime}$. It consists of a $1 / 4 \AA$ band pass birefringent filter, an SMD $1024 \times 1024$ 12-bit CCD camera and polarization analyzer. Each data set consists of four images: a 6103 Å filtergram (Stokes-I), a line-of-sight magnetogram (Stokes-V) and the transverse magnetogram (Stokes-U and $-\mathrm{Q}$ ). We rebin the camera to the $512 \times 512$ mode to increase the sensitivity of the magnetograms.

The polarization analyzer includes two nematic liquid crystal variable retarders, whose retardance can be controlled with an applied voltage, to select a particular polarization state (6103 ̊ filtergram, Stokes $\mathrm{V}, \mathrm{Q}$, or $\mathrm{U}$ ) by converting the desired input polarization set into an orthogonal set of linear polarizations. 
A single ferroelectric liquid crystal, which is a fixed retarder whose rotation angle can be selected to be either 0 or 45 degree, acts as the system's fast modulator. This ferroelectric crystal, working with a fixed linear polarizer, is used to select one of the orthogonal linear polarization components. Light is then fed through the birefringent filter, and finally imaged onto the CCD camera. The exposure is typically $30 \mathrm{~ms}$, and images are taken at a rate of approximately 12 frames $\mathrm{s}^{-1}$.

A distinct characteristic of the AR evolution is the appearance of sheared emerging flux regions (EFRs) which were current-carrying. They emerged successively and grew along the main magnetic neutral line of the AR. In Figure 1, a time sequence of vector magnetograms, obtained from Huairou Solar Observing Station (HSOS) of National Astronomical Observatories of Chinese Academy of Sciences, is shown. These EFRs make the AR grown rapidly since January 13. On the figure four EFRs are marked by brackets. A very key characteristic of these EFRs is the appearance of a bundle of enhanced transverse fields which are connecting the growing magnetic flux of opposite polarity. The emergence of the four EFRs can be traced since January 13 until January 18. It is interesting that all these four EFRs were growing along the main magnetic neutral line, and separating in roughly the opposite direction perpendicular to the the main magnetic axis of the AR. It is noticed that the EFR marked close to the center of the magnetogram at 01:20 UT of January 15 showed opposite sense of twisting, i.e., opposite sign of magnetic shear or current helicity in comparing with other EFRs. The co-existence of opposite helicity in new EFRs made the 3D magnetic configuration more complicated. Later from January 16, another new EFR appeared in the area of negative flux to the north of the magnetic neutral line (see the growing positive magnetic flux).

The effects of these EFRs are presented in several perspectives: (1) The magnetic neutral line was largely elongated. From 00:32 UT of January 13 to 16:51 UT of January 15, the neutral line was elongated from $120 \mathrm{Mm}$ to $260 \mathrm{Mm}$; (2) Interleaving opposite polarities and multiple neutral lines appeared in the center of the AR; (3) Strong magnetic shear developed along this elongated neutral line in roughly the East-West direction. The net results of these EFRs appear to be equivalent to a widely separated 'EFR' as indicated by thick arrows in the magnetogram at 03:58 UT of January 16. Overall speaking, the opposite polarities of newly emerged flux separated at a velocities of $0.5-0.7 \mathrm{~km} \mathrm{~s}^{-1}$ in the observed interval.

The central meridian passage of AR 10720 took place On January 15. An X-ray flare with significance of X2.6 appeared at 22:25 UT in the AR. This flare is one of a significant flares associated with a fullhalo CME and serves as a typical active event for careful exemplification. From 16:51 to 23:47 UT BBSO obtained 262 sets of high quality vector magnetograms with 20 Gauss sensitivity in line-of-sight magnetograms, and 150 Gauss in transverse magnetograms (Spirocks 2005). These observations fully confirmed the evolutionary characteristics of the vector magnetic fields in the AR which were revealed by HSOS. In Figure 2, vector field evolution in 7-hour interval is shown in details. The consistence in flux distribution and field azimuth is impressive between HSOS and BBSO magnetograms. The well-known 180 degree ambiguity in the observed field azimuth is resolved by potential and constant- $\alpha$ force-free field assumptions. A few empirical corrections, based on the history of flux emergence and the continuity in the observed azimuth, are made after removing the ambiguity in the above objective ways.

There is no doubt that the topology skeleton in the magnetized plasma of the AR is determined by the magnetic field distribution and evolution in the photosphere. BBSO vector magnetograms were obtained at much better seeing and with a cadence of 1-2 minutes. In addition, the BBSO vector magnetograms covered the whole interval of the major flare/CME on January 15 from pre-status to full-recovery phases. Therefore the BBSO magnetograms provide a rare opportunity to observationally determine the magnetic skeleton of a solar AR, and to identify the characteristics of the skeleton evolution in the course of explosive energy release in solar activity.

\section{METHODS}

We use the observed vector magnetograms of BBSO as boundary conditions, employing a quasi-linear force-free code (Wang et al. 2001), we reconstruct the 3D magnetic fields of AR 10720 first. For the magnetograms which we selected are very near to the center of the solar disk, we can use them directly without solar rotation correction. Due to the influence of the stray light, there exist areas of polarization saturation, which appeared as 'big holes' in the middle of the strong sunspots in longitudinal magnetograms. We use the MDI longitudinal magnetogram at the nearest time to fulfill these 'holes'. 


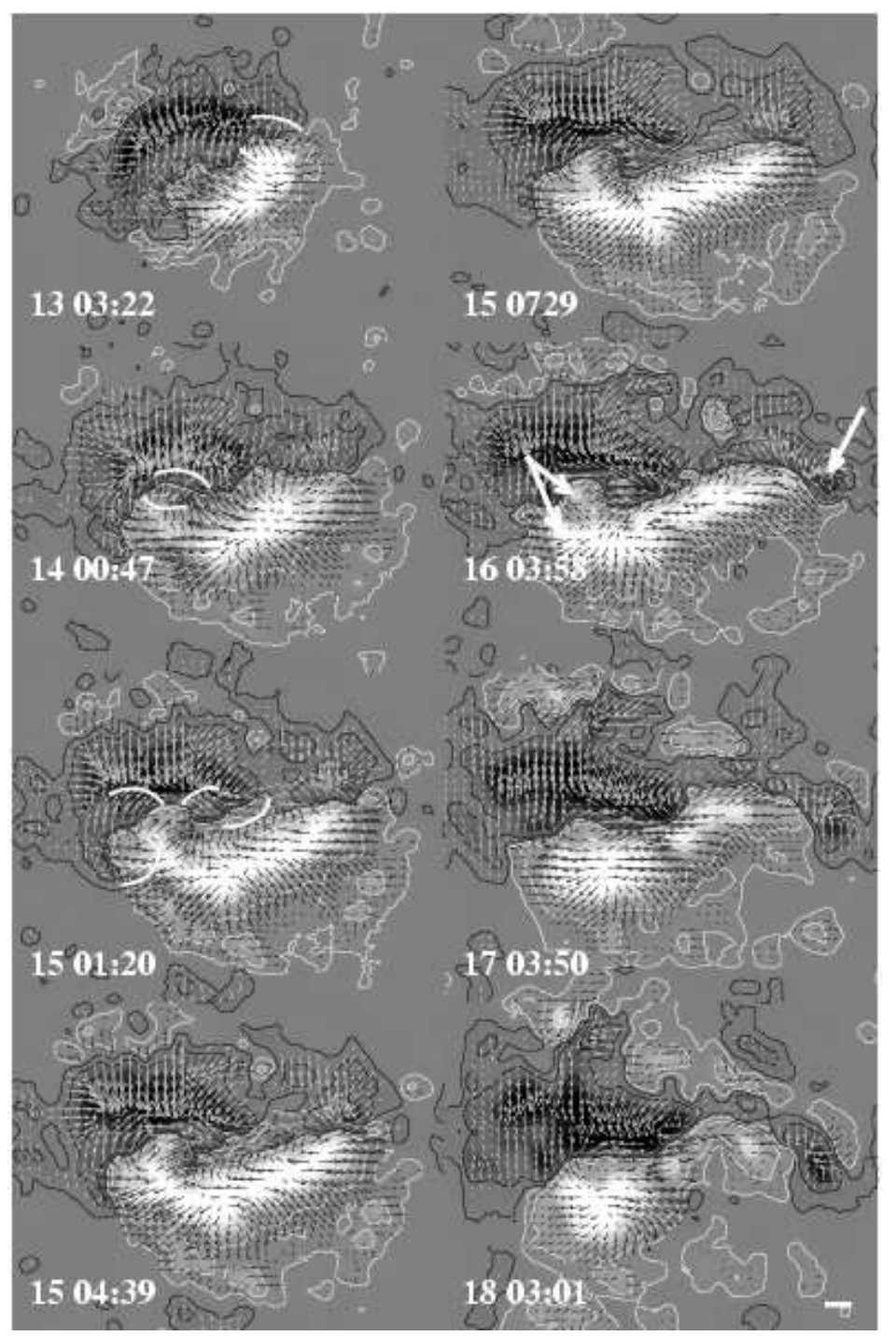

Fig. 1 Time sequence of Huairou vector magnetograms. The line of sight component is shown by images scaled from -1500 to $1500 \mathrm{G}$ and contours with levels of $\pm 50,250,500,1000,1500 \mathrm{G}$. The transverse components are shown by short arrows with length proportional to the field strength and direction indicated by the arrows. Four key EFRs are marked in the magnetograms by brackets. Each EFR is characterized by a bundle of enhanced transverse fields and two growing footpoints of opposite polarity. At 03:08 UT of January 16, three thick arrows indicate the concentrated magnetic flux of opposite polarity which come from the successive EFRs from the interval of January 13 to 16 . The scale bar in the lower-bottom corner denotes 20.0 arcsec.

The size of extrapolation box is $250 \times 250 \times 150$ in bins. Each bin corresponds to $2.1^{\prime \prime}$. The observed magnetogram embed in the middle of $150 \times 150$ as shown in Figure 4 of this paper.

We search for nulls in every cell of the box with Poincaré index (Greene 1992; Zhao et al. 2005) after we obtain the 3D vector fields at each node in the AR. This method in 2D conditions have been used on vector magnetograms by Wang \& Wang (1996). In 3D condition, we get at first the magnetic vectors in eight vertex of each cell, then we normalize these vectors and translate these images of vectors to a unit 


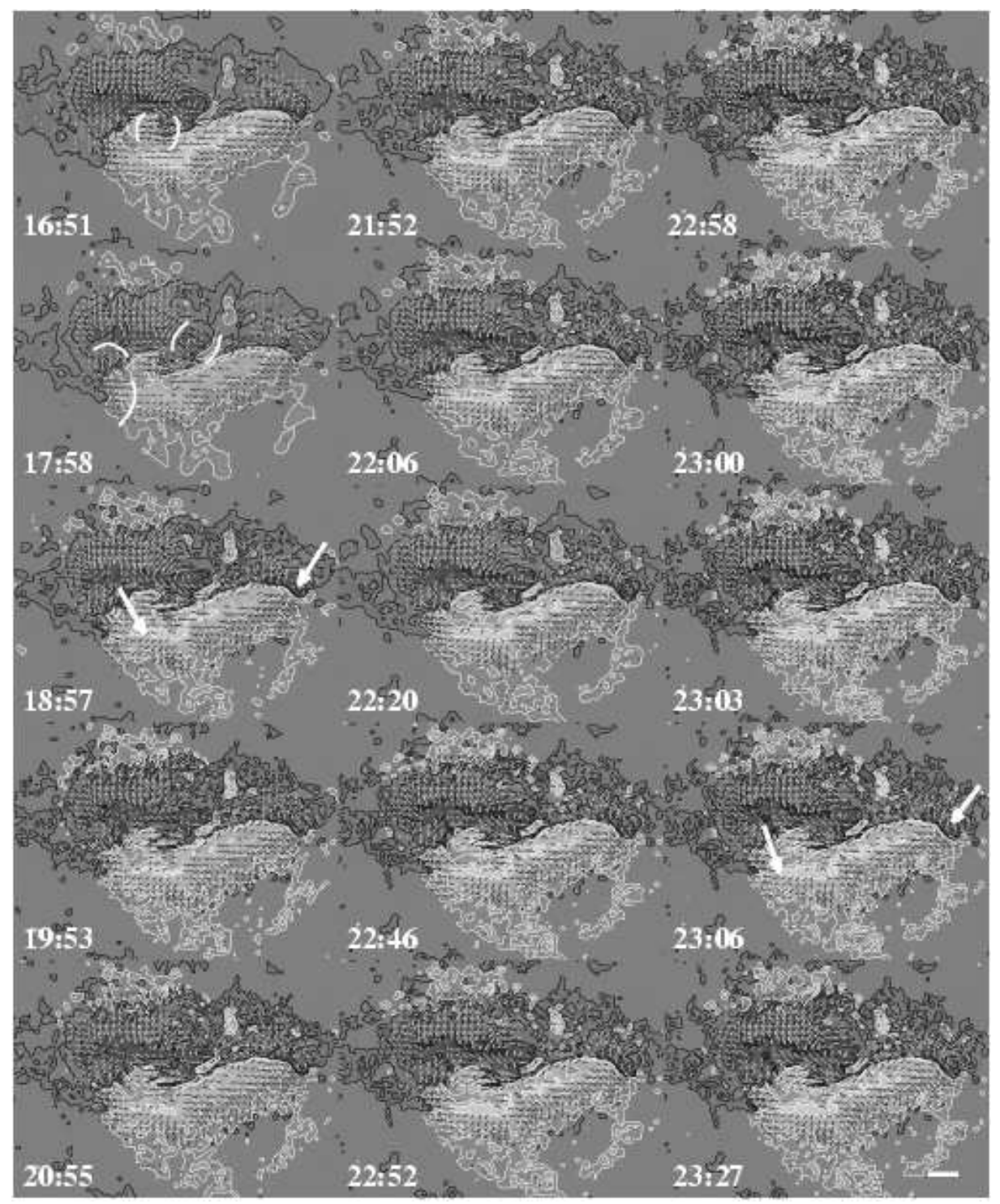

Fig. 2 Time sequence of BBSO magnetograms which are shown by the same style as in Figure 1. Three EFRs in continuous growing are marked in the magnetograms by brackets. Two sets of thick arrows indicate the concentrated magnetic flux of opposite polarity which come from the successive EFRs since January 13. Note, the opposite polarities of the equivalent EFR are in continuous growing and separating. The scale bar denotes 25.0 arcsec.

sphere. The cubic surface of each cell can naturally be separated into 12 triangles (see fig. 2 in Zhao et al. 2005), every triangle map to a spherical triangle in the unit sphere. By integrating these twelve spherical triangles and dividing the integral by $4 \pi$, we obtain the Poincaré index in practice. A little different from 2D conditions (Wang \& Wang 1996), if a cell include a null point, the index should be equal to 1 or -1 , if not, 0 . The nulls whose initial positions are outside the middle $150 \times 150 \times 50$ bins or lower than 1 grid are ignored, for extrapolation in those regions is not very credible, or foot points of the field lines through this region overstep the observed view. We extend the extrapolation scale for the sake of pursuing some nulls' evolution. 


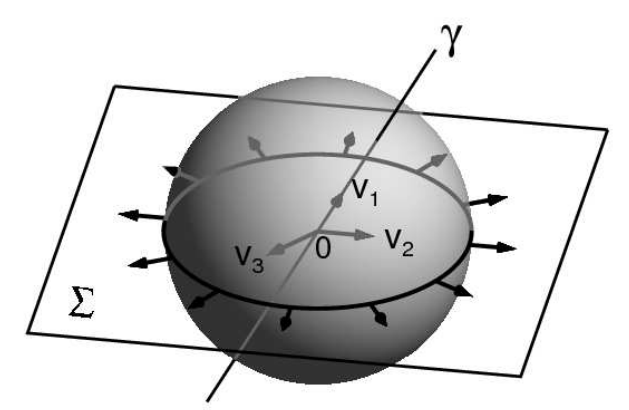

Fig. 3 Illustrate the structures near to a (B type) null. A unit sphere will wrap a cell which include a null. The $\gamma$ line and the $\Sigma$ surface of the null intersect at it. They cross the sphere at two points and a circle, in which the vectors parallel to the directions of the $\gamma$ line and the $\Sigma$ surface, respectively. These directions are determined by the three eigenvectors, $\mathbf{v}_{1}, \mathbf{v}_{2}, \mathbf{v}_{3}$, of the matrix $\delta \mathbf{B}$.

In order to draw out the magnetic skeleton above the active region, we calculate the field's Jacobian matrix $\delta \mathbf{B}$ in each null-point which has been identified. The matrix $\delta \mathbf{B}$ has three eigenvalues and three eigenvectors, says, $\lambda_{1}, \lambda_{2}, \lambda_{3}$ and $\mathbf{v}_{1}, \mathbf{v}_{2}, \mathbf{v}_{3}$, respectively. When the index is not equal to zero, the null would be a 3D isolated singular point. This means the null will not fall to a $2 \mathrm{D}$ null (according to the definition of 3D isolated singular point), hence the matrix $\delta \mathbf{B}$ is regular and none of the eigenvalue is zero. The trace of $\delta \mathbf{B}$ is equal to the sum of the three eigenvalues. On the other hand, the trace can be presented by $\nabla \bullet \mathbf{B}$ which is zero in a magnetic field. The three eigenvalues may be all real numbers or one is real number, the other two are conjugated complexes. However, the real parts of them should be $(+--)$ or $(-++)$ (because the sum of the three real parts is zero), if they are all real numbers, the null is type $A$ or type $B$, if two of them are complexes, the null is type $A_{s}$ or type $B_{s}$ (Cowley 1973; Lau \& Finn 1990). One eigenvector ( $\mathbf{v}_{1}$ for example), according to the eigenvalue which is the only positive or negative, points out the direction of $\gamma$ line (or spine) and the other two $\left(\mathbf{v}_{2}\right.$ and $\mathbf{v}_{3}$ ) determine $\Sigma$ surface (or fan) near the null-point (Fukao et al. 1975; Lau \& Finn 1990). In practice, we select a small sphere (radius equals to one grid unit) including each null (cause the sphere is big enough to include the cell). The spines and fan should intersect the sphere at two points and a circle respectively (Fig. 3). The vectors in the two points would parallel to the spine eigenvector and the vectors on the circle would parallel to the plane determined by the two fan eigenvectors. Therefore we can know which point on the sphere belongs to the intersection of the line of spines or fan by check the directions of the vectors on the sphere whether parallel to the directions of spine or fan. We draw lines of fan departing from the sphere along the circle (denote $l(x)$, where $x$ is belong to the circle), if a infinite little distance $\epsilon$ in point $x$ make the other endpoints of $l(x-\epsilon)$ and $l(x+\epsilon)$ sharply depart from each other, then there must be a separator line $l_{s}(x)$ start from $x$. When the two endpoints of a separator are both null points, the separator is called a null-null line. Finally we obtain the magnetic skeleton which is composed of nulls, spines fans and separators.

\section{TOPOLOGY SKELETON OF AR 10720}

In brief summary using the observed vector magnetograms as boundary conditions, we first reconstruct the 3D magnetic fields in the AR's atmosphere with a quasi-linear force-free code (Wang et al. 2001); then we identify the 3D magnetic nulls by a differential geometry method (Greene 1992; Zhao et al. 2005) in the extrapolated vector fields; finally we determine the magnetic skeleton by connecting the nulls, spines, fans and separators. We compare the skeletons with flare ribbons and EIT light structures, also we examine the skeleton evolution from the derived time-sequence of topologies. 
Table 1 Information of nulls at 21:52 UT.We employ the coordinate in Figure 1 to present the nulls' positions. $\triangle B=100 \mathrm{G}(0.01 \mathrm{~T}), \mathrm{L}=1$ unit grid $(1.523 \mathrm{Mm}) .|\langle\triangle B\rangle /\langle L\rangle|$ denotes the average error in the derivatives of the observed magnetic field.

\begin{tabular}{ccccc}
\hline No & Type & $\begin{array}{c}\text { Position } \\
(\mathrm{Mm})\end{array}$ & $\begin{array}{c}\text { Eigenvalues } \\
\left(10^{-10} \mathrm{~T} \mathrm{~m}^{-1}\right)\end{array}$ & $\frac{|\nabla \bullet B|}{\langle\Delta B\rangle /\langle L\rangle \mid}$ \\
\hline I & $A$ & $(111,222,55)$ & $0.967,-0.115,-0.194$ & 0.010 \\
II & $B$ & $(172,215,49)$ & $-0.167,0.080,0.592$ & 0.008 \\
III & $A$ & $(207,117,11)$ & $1.847,-0.523,-0.814$ & 0.008 \\
IV & $A_{s}$ & $(177,125,47)$ & $0.308,-0.038 \pm 0.735 i$ & 0.004 \\
V & $B$ & $(148,120,9)$ & $-3.120,1.079,4.405$ & 0.036 \\
VI & $A$ & $(140,126,3)$ & $4.795,-2.620,-4.738$ & 0.039 \\
\hline
\end{tabular}

\subsection{Determined Topology Skeleton}

The determined skeleton at 21:25 UT of January 15 basically consists of six nulls (see Fig. 4). Each null and its associated $\gamma$-lines (spines) and $\Sigma$-surface (fan) are referred to as a topology, and numbered as Topologies I, II, ..., VI, respectively. Their locations, eigenvalues, and divergence of magnetic vector in term of observational errors are listed in Table 1. Majority of the magnetic lines of force (see the lower panel of Fig. 4) which anchored in the photosphere are bounded by a combination of fans and spines of the Topologies I, II, III, and V, forming AR's magnetized atmosphere. Topology IV is embedded in the middle of the atmosphere.

Amongst the six, Null IV is of $A_{s}$ type, i.e., a spiraling-in null. It locates at an altitude of $47 \mathrm{Mm}$. For the first time we have identified a spiral null in the Sun's corona of an AR, based on observations. The spine of $A_{s}$ null lies above the whole length of the magnetic neutral line, along which the 'sheared EFRs' emerged (see Figs. 1 and 2). Around the spine of the $A_{s}$ null the magnetic lines of force twisted, forming a magnetic wreath shaped like trumpet shell, i.e., a complicated flux rope structure. Around the spine of the $A_{s}$ null the magnetic lines of force twisted, forming a magnetic wreath shaped like trumpet shell, i.e., a complicated flux rope structure. This structure obviously can be a kind of physical entities of flux rope, a concept commonly used in solar physics, but a rope structure may not have an $A_{s}$ or $B_{s}$ null in its center. The Fan V and a part of the Fan III join to the twisted lines of force. In fact, the rotated lines of force which consist the loop-like structure just boundary with Fan III, Fan IV and Fan V. The electric current density flowing along the spine is as high as $3 \times 10^{-4} \mathrm{~A} \mathrm{~m}^{-2}$ at such an altitude in the corona. It is, at least, two times higher than that in surroundings, indicating a concentration of free magnetic energy. The currents also flew in the fan surface, which resulted in a tilt angle of 75 degrees between the spine and fan.

The main loop-like structure is consisted by the Topology IV and Topology V, which derived from Null IV and Null V, respectively. As we know, the complicated structure centered with a null-null pairs which include one or two spiral nulls is only described by numerical simulations based on plasma kinetic approaches (Büchner 1999; Cai et al. 2006). For demonstrating the $A_{s}-B$ null pairs' structure more clearly, we simplified the structure in ideal condition by a cartoon in Figure 5 . The $\Sigma_{A_{s}}$ intersects the $\Sigma_{B}$ with $A_{s}-B$ null-null line, the separator, and the $\Sigma_{A_{s}}, \Sigma_{B}$ are semi-infinite sheets bounded by the $\gamma_{B}, \gamma_{A_{s}}$, respectively. The field lines on $\Sigma_{A_{s}}$ twisted into the $A_{s}$ null, and $\Sigma_{B}$ rolls over onto $\gamma_{A_{s}}$ (note that the part of the field lines on the $\Sigma_{B}$ asymptotically approach the $\gamma_{A_{s}}$ but can not touch it). The field lines on the $\Sigma_{B}$ which asymptotically approach the $\gamma_{A_{s}}$ and the field lines on the $\Sigma_{A_{s}}$ compose a loop-like structure. We should point out that in Figure 4 the Fan IV, a $\Sigma_{A_{s}}$ surface, is not a semi-infinite sheet because it is bounded not only by Spine $\mathrm{V}$, a $\gamma_{B}$ line, but also by photosphere.

The six nulls are connected by a net of null-null lines, the so-called separators, upon which the frozenin magnetized plasma of four individual topologies closely contacts and interacts one another. The adjacent null pairs in the separators are all of $A-B$ or $A_{s}-B$ type. These fans and their intersection, the separators, separate the magnetized atmosphere into different domains in which the lines of force have the same connectivity. The net of separators bridging distinct topologies goes through the magnetized atmosphere. This fact hints the characteristic of 3D magnetic reconnection in the AR. As the reconnection could take place 
successively or simultaneously along the whole separator net, the magnetic energy release during the reconnection in the AR should be global, fast and explosive in nature (Priest et al. 1997; Priest \& Forbes 2000; Parnell 2007).

\subsection{Spacial Relations with Flare Ribbons and Brightening EUV Loops}

It is interesting to see that the cross-sections of spans of the lower Topologies III, V, and VI are coincided with the main parts of the flare ribbons (see lower panel of Fig. 4) as described by many authors previously (see Démoulin et al. 1997). The south-west piece of flare ribbons seemed to apparently avoid from all topology structures, but, in fact, was clearly connected to the fans of Null V by a set of magnetic arcades straddling the magnetic neutral line.

Such complicated rope structures organized by a spiral null were never anticipated until we draw out the topology skeleton. The Fans IV and V intersect at the $A_{s}-B$ null-null line, the separator; they are bounded asymptotically by Spines V and IV, respectively. The field lines on Fan IV twisted into the Null IV, the $A_{s}$ type null, and Fan V rolls over onto Spine IV. The field lines on the Fan V, which asymptotically approach the Spine IV, and field lines on the Fan VI and a fraction of Fan III compose the frame of the magnetic wreath. It is remarkable that the magnetic lines of force on both sides of Fan IV have opposite twisting, i.e., the magnetic helicity in the rope-like structure does not keep the same sign. Although the current data analysis does not reveal the details of associated 3D reconnection, we witness the close similarity between the 'magnetic wreath' and EUV rope-like structures, brightened in the course of the flare/CME (see Fig. 6). The EUV brightening took place in a twisting structure similar to the magnetic lines of force in the magnetic wreath, said, the general shape and sharp boundaries of EUV structure well resembled the magnetic wreath. This implies the close correlation between the energy release and topology structure.

\subsection{Skeleton Evolution}

Based on the time sequence of observed vector magnetograms, we further deduced the skeleton evolution from 21:52 to 23:47 UT (see Fig. 7), covering the interval from pre-status to recover-phase of the flare/CME. In the course of the activity event, the topology skeleton showed obvious evolution, which could be categorized into slowly systematic evolutions and explosive eruption. One of the key systematic evolutions is elevation of the spiral null together with its spines. The spiral null moved up more than $26 \mathrm{Mm}$ during the studied interval. At the same time, the magnetic wreath grew. In accordance, there is also an expansion of Topology I. In the expansion, Null I moved northward more than 50 Mm from 21:52 to 22:52 UT. The dramatic evolution is likely a response to the rapid growth of the 'sheared EFRs'. During this interval the apparent separation speed of opposite polarity flux of the EFRs was about $1.0 \mathrm{~km} \mathrm{~s}^{-1}$. The fast and continuous separation of opposite polarities of the new emerging flux can be seen clearly from the south-west movement of the negative flux patch (already grew to a sunspot) indicated by a thick arrow in Figure 2.

In the course of the systematic evolution two topology eruptions took place in association with the flareCME development. Null II and its topology erupted right before the flare and shortly after the initiation of the halo CME at 22:36 UT, which passed into LASCO C2 field of view at 23:06 UT with a speed of more than $2800 \mathrm{~km} \mathrm{~s}^{-1}$. The second topology eruption appeared at the maximum phase of the flare and manifested as the disappearance of Null I and its topology. The separators connecting the Nulls I and II disappeared in the two topology eruptions, which is indicative of the vigorous 3D magnetic reconnection along these null-null lines in the flare/CME event. However, we may not exclude a possibility that the two 'disappeared' topologies might simply move outside the calculation domain. To test this possibility, we have extended our calculations to more than $200 \mathrm{Mm}$ above the photosphere, but found no hints of their existence in the higher corona. During the skeleton evolution, the three lower nulls, Nulls III, V, and VI, and their topologies remained, keeping the spatial relations with flare ribbons unchanged.

\section{DISCUSSION}

There may appear some uncertainties in determination of magnetic skeleton, which come mainly from the $3 \mathrm{D}$ reconstruction of magnetic fields. We have examined the reliability of the approaches in this work by artificially introducing random noises in the observed magnetograms, and found that the basic skeletons that we determined are re-producible. 
It is well-known that with the same boundary condition different algebra and codes would give different force-free solutions, i.e., the extrapolation of force-free fields from observations is not unique (Seehafer, 1978). We have tested the robusticity of our findings by using different force-free codes. First of all, the potential and linear force-free field extrapolation models and methods (Alissandrakis 1981; Seehafer, 1978; Chiu and Hilton, 1977) could not well reproduce the skeleton which we determined. The potential and exactly constant- $\alpha$ force-free extrapolations were imbecilic in finding spiral nulls. The reason is that opposite helicity signs maintain in the magnetic structures when there is a spiral null, and the co-existence of opposite sign helicity, hence the $\alpha$ value, is not allowed by potential or linear force-free models.

These new skeletons reconstructed by other extrapolation codes have rare relation with the flare ribbons and it revealed that the potential and exactly constant- $\alpha$ force-free extrapolations were imbecilic in finding spiral nulls and a right transverse field is necessary for reconstruction based on a complicated vector magnetogram with strong shearing.

For severely twisted or sheared magnetic topology, the codes based on boundary element method (BEM) which are developed by Wang et al. (2001) and Yan \& Sakurai (2000) may have some advantage. In the comparison based on an analytical model (Low \& Lou 1990), the BEM (Liu et al. 2002) performed better than potential and constant $\alpha$ method and has less disparity nearer the center of the extrapolation box (Schrijver et al. 2006).

We have been trying to use other non-linear force-free models (Wheatland et al. 2000; Wheatland 2006, 2007; Song et al. 2006) to examine if the current results can be repeated by other code independently. More careful work still needs to be done before drawing definitive conclusions. While the complicated topology skeleton determined in this study has not been reproduced by other potential and linear force-free codes so far, the reality of the determined skeleton can only be hinted by its comparison with observations of brightness structures in $\mathrm{H} \alpha$, EUV, and X-ray wavebands. As shown by Figures 4, 6 and 7, the $\mathrm{H} \alpha$ flare ribbons are closely associated with the lower nulls and their topologies, while the higher nulls (including the spiral null) and their topologies are more closely correlated to the CME onset. Because our determined topology skeleton does match some of the activity structures in both spatial and temporal domains, we believe that our determination represents, at least, some aspects of the real 3D topology peculiarity.

More active regions are under consideration and a few different force-free codes are undertaken as future efforts to observationally determine the topology skeleton of magnetic fields in active regions.

In the case that the 3D vector field measurements, either by in situ or remote-sensing, are available, our method to determine the 3D nulls is robust and ready to be used.

\section{CONCLUSION}

Based on the time sequence of observed vector magnetograms with high resolution and sensitivity, we have determined the 3D magnetic skeleton and its evolution in a solar AR. We have identified, for the first time, the spiral magnetic null in the Sun's corona. Extremely complicated rope structures, the magnetic wreath, associated with the spiral null appear to be the central ingredient of the magnetic skeleton. The determined skeleton has close tempo-spatial associations with solar explosive activity. The skeleton evolves in responds to the vector field evolution in the solar photosphere. We have clearly uncovered two topology eruptions in the course of the flare/CME. The complexity of observed magnetic skeleton and its evolution in solar active atmosphere was not previously reported in the literature.

Although spiral nulls are predicted in theoretical approaches (Lau \& Finn 1990; Parnell et al. 1996), they have never been discovered in solar observations. However, their appearance and role in 3D magnetic reconnection have been clearly described by numerical simulations based on plasma kinetic approaches (Büchner 1999; Cai et al. 2006). Our observations fully support the theoretical predictions and numerical simulations. For many years solar astronomers have hypothesized the common existence of flux ropes in Sun's corona. The flux rope concept becomes a central element in CME modelling in solar physics. However, the observational identifications were not available in the literature. Different authors use the same scientific term, 'flux rope', but refer to different physical entities in observations (Hudson \& Cliver 2001). Here we propose that the magnetic topology of a spiral null in the corona, e.g., the magnetic wreath, serves, at least, one type of the real flux ropes. Flux ropes are likely to have spiral nulls inside in reality. Their magnetic structures appear to be more complicated than that one has thought. Within a flux rope opposite signs of magnetic helicity can be kept in association with topology peculiarity. The magnetic reconnection 
between opposite helicity flux may result in explosive release of more free magnetic energy (Wang et al. 2004)

Acknowledgements This work was supported by National Basic Research Program of China (2006CB806303) and National Natural Science Foundations of China (G10573025 and 40674081). We should thank for the help of H. N. Wang, E. R. Priest, M. S. Wheatland and M. T. Song.

\section{References}

Ai G., Hu Y., 1986, Publ. Beijing Astron. Obs., 8, 1

Alissandrakis C. E., 1981, A\&A, 100, 197

Aulanier G., DeLuca E. E., Antiochos S. K. et al., 2000, ApJ, 540, 1126

Brown D. S., Priest E. R., 2001, A\&A, 367, 339

Büchner J., 1999, Astrophys. and Space Sci., 264, 25

Bungey T. N., Titov V. S., Priest E. R., 1996, A\&A, 308, 223

Cai D., Nishikawa K., Lembege B., 2006, Plasma Phys. Control. Fusion, 48, B123-B135

Chiu Y. T., Hilton H. H., 1977, ApJ, 212, 873

Cowley S. W. H., 1973, Radio Sci., 8, 903

Démoulin P., Henoux J. C., Mandrini C. H., 1992, Solar Phys., 139, 105

Démoulin P., Henoux J. C., Priest E. R., Mandrini C. H., 1996, A\&A, 308, 643

Démoulin P., Bagalá L. G., Mandrini C. H. et al., 1997, A\&A, 325, 305

Filippov B., 1999, Solar Phys., 185, 297

Fletcher L. et al., 2001, ApJ, 554, 451

Fukao S., Masayuki U., Takao T., 1975, Rep. Ionos. Space Res. Jpn., 29, 133

Giowanelli R. G., 1946, Nature, 158, 81

Gorbachev V. S., Somov B. V., 1988, Solar Phys., 117, 77

Greene J. M., 1992, J. Comp. Phys., 98, 194

Hudson H. S., Cliver E. W., 2001, J. Geophys. Res., 106, 25199

Lau Y. T., 1993, Solar Phys., 148, 301

Lau Y. T., Finn J. M., 1990, ApJ, 350, 672

Li H., Schmieder B., Aulanier G., Berlicki A., 2006, Solar Phys., 237, 85

Liu Y., Zhao X. P., Hoeksema J. T. et al., 2002, Solar Phys., 206, 333

Longcope D. W., 2005, Living Rev. Solar Phys., 2, 7

Longcope D. W., Klapper I., 2002, ApJ, 579, 468

Low B. C., 1987, ApJ, 323, 358

Low B. C., Lou Y. Q., 1990, ApJ, 352, 343

Low B. C., Wolfson R., 1988, ApJ, 324, 574

Maia D. et al., 2003, A\&A, 405, 313

Parnell C. E., 2007, Mem. S.A.It., 78, 229

Parnell C. E., Smith J. M., Neukirch T., Priest E. R., 1996,J. Plasmas Phys., 3, 759

Priest E. R., Bungey T. N., Titov V. S., 1997, Geophys. Astrophys. Fluid Dyn., 84, 127

Priest E. R., Démoulin P., 1995, J. Geophys. Res., 100, 23,443

Priest E., Forbes T., 2000, Magnetic reconnection: MHD theory and applications, Cambridge: Cambridge Univ. Press

Schrijver C. J., Derosa M. L., Metcalf T. R. et al., 2006, Solar Phys., 235, 161

Seehafer N., 1978, Solar Phys., 58, 215

Seehafer N., 1986, Solar Phys., 105, 223

Spirocks T., 2005, thesis, New Jersey Institute of Technology

Song M. T., Fang C., Tang Y. H. et al., 2006, ApJ, 649,1084

Sweet P. A., 1958, IAU symposium, 6, 123

Titov V. S., Hornig G., Démoulin P., 2002, J. Geophys. Res., 107, 1164

Titov V. S., Priest E. R., Démoulin P., 1993, A\&A, 276, 564

Wang H. N., Yan Y. H., Sakurai T., 2001, Solar Phys., 201, 323

Wang H. N., Wnag J. X., 1996, A\&A, 313, 285

Wang J. X., Shi Z. X., Wang H. N., Lü Y. P., 1996, ApJ, 456, 861

Wang J. X., Zhou G. P., Zhang J., 2004, ApJ, 615, 1021 
Wheatland M. S., Sturrock P. A., Roumeliotis G., 2000, ApJ, 540, 1150

Wheatland M. S., 2006, Solar Phys., 238, 29

Wheatland M. S., 2007, Solar Phys., submitted

Xiao C. J. et al., 2006, Nature Phys., 2, 478, DOI: 10.1038/nphys342

Yan Y. H., Sakurai T., 2000, Solar Phys., 195, 89

Zhao H., Wang J. X., Zhang J., Xiao C. J., 2005, Chin. J. Astron. Astrophys. (ChJAA), 5, 443 


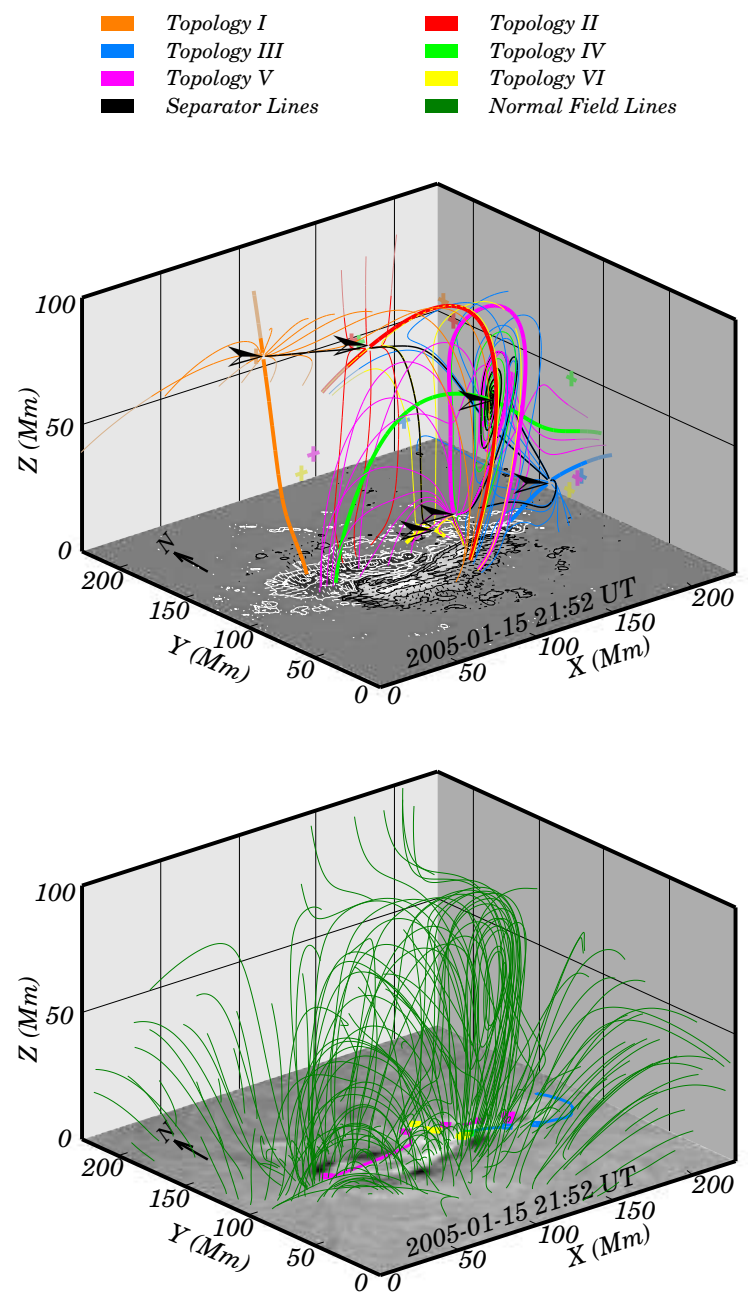

Fig. 4 The 3D magnetic structures and determined topology skeleton of AR10720. The magnetic lines of force extrapolated by a quasi-linear force-free code based on the observed BBSO vector magnetogram at 21:52 UT of January 15, 2005 is shown in lower panel; while the magnetic skeleton determined from the extrapolated 3D fields is shown in upper panel. The vector magnetogram is displayed as the background of upper one, in which the line-of-sight flux density is shown by grey map with contours of 200, 700, 1200 Gauss and bright (dark) color for positive (negative) flux; the transverse magnetogram is shown by short arrows with length representing the field strength. Each null and its spines and fan is referred to as a topology, and numbered from 'I' to 'VI'. The positions of the nulls are indicated by arrows while their projections in the two vertical planes are marked by symbols '+'. Different topologies are drawn with distinct colors. Thick lines are spines while thin lines are magnetic lines on the fans. A BBSO $\mathrm{H} \alpha$ filtergram is shown as background of panel lower one, in which the intersections of fans with chromosphere are drawn to show their correlation with flare ribbons. 


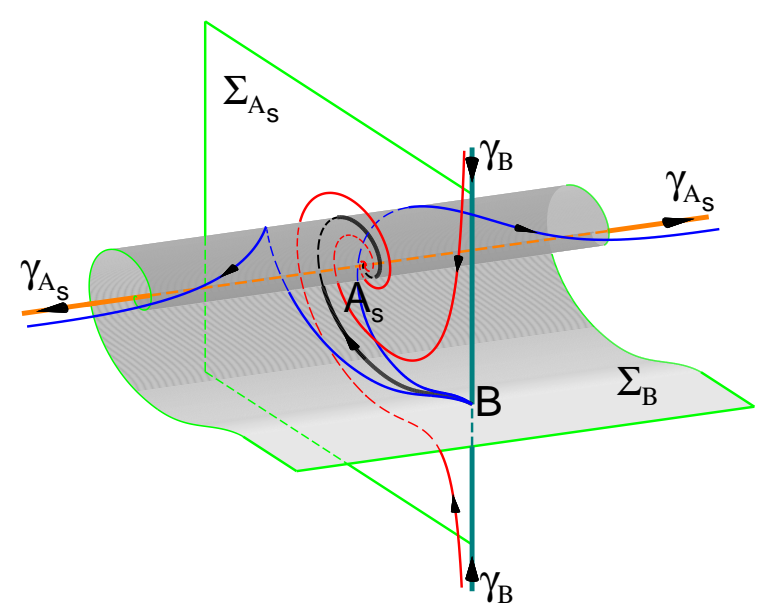

Fig. 5 A cartoon used to simplify the topology structure of the $A_{s}-B$ null-null pair. Orange and celadon lines are $\gamma_{A_{s}}$ and $\gamma_{B}$ lines respectively. Red and blue lines present the fields lines on $\Sigma_{A_{s}}$ and $\Sigma_{B}$. The black line at which $\Sigma_{A_{s}}$ and $\Sigma_{B}$ intersect is the $A_{s}-B$ null-null line. Note, the twisting sense of magnetic lines of force on both sides of the $\Sigma_{A_{s}}$ is opposite.

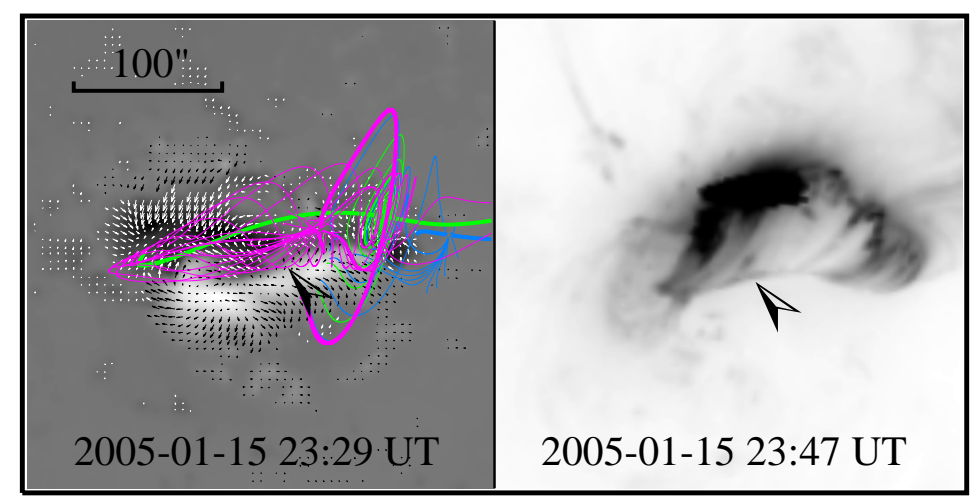

Fig. 6 Comparison of the magnetic wreath associated with the spiral null (left panel) and EUV brightening structure in the late phase of the X2.6 flare (right panel). Left: The topology skeleton is superposed above a BBSO vector magnetogram at 23:29 UT. Right: The general appearance of the EUV brightening structures is shown by the reversed color table in which the brightest structures are darkest. The EUV structures resemble the topology skeleton, particularly the low sharp boundary seems to coincide with the low boundary of the wreathed $\Sigma$ surfaces of Nulls III and $\mathrm{V}$ (the two boundaries are pointed by arrows). 

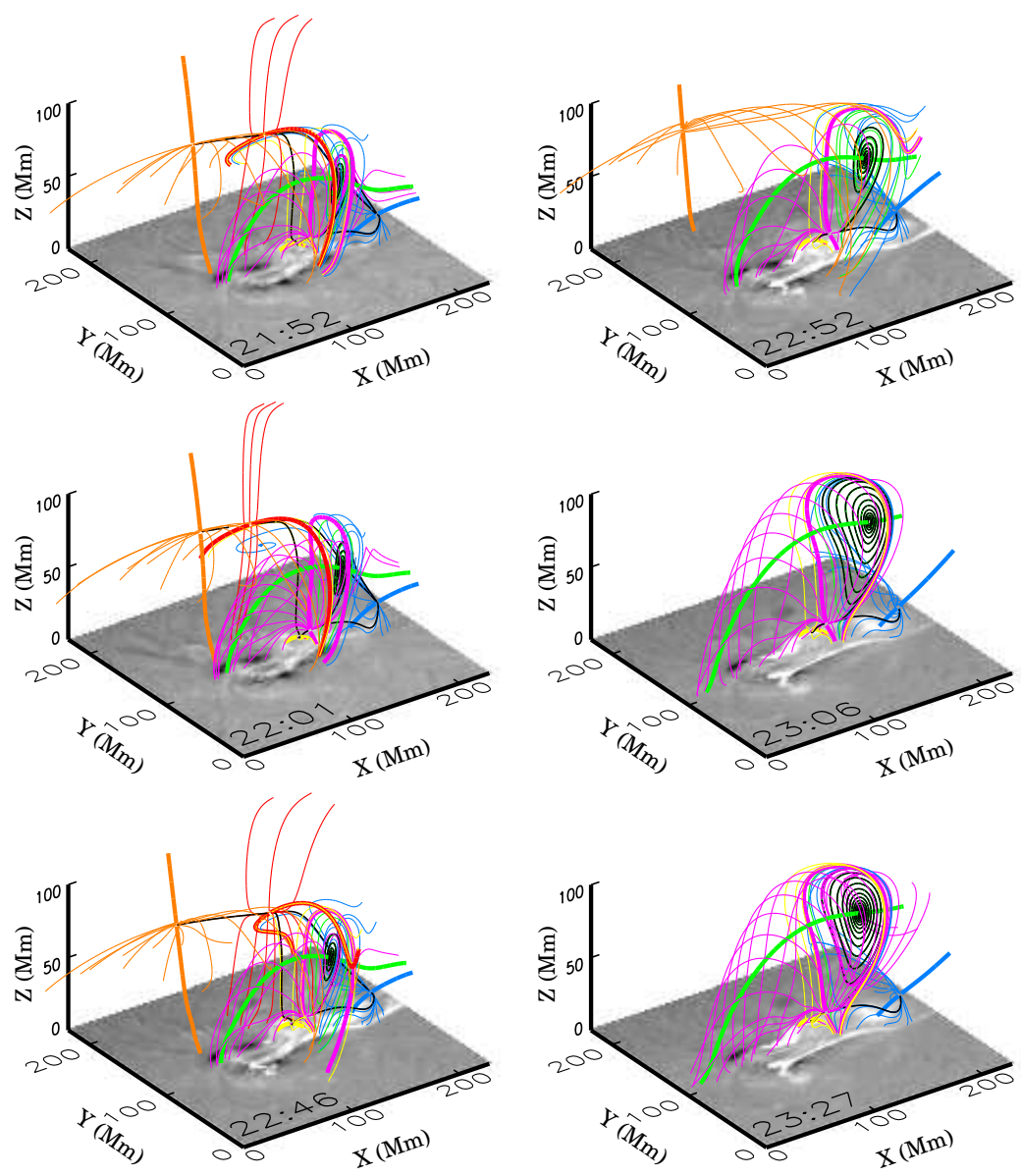

Fig. 7 Skeleton evolution in the interval from pre-state to the recovery phase of the flare/CME. Null II and its topology disappeared between 22:46 to 22:52 UT, in which the CME initiated from the solar disk; while the null I and its topology disappeared between 23:03 to 23:06 UT at the maximum phase of the flare. The drawing style of this figure is same as Fig. 1. The elevation of null IV and the mounting up of magnetic wreath are clearly shown in the time-sequence of skeleton evolution. 